\title{
Toward millions of laser pulses with pyrromethene- and perylene-doped xerogels
}

\author{
Mohammed Faloss, Michael Canva, Patrick Georges, Alain Brun, Frédéric Chaput, \\ and Jean-Pierre Boilot
}

Significant improvements have been obtained for solid-state dye lasers with doped xerogels. By using longitudinal pumping with a frequency-doubled $Q$-switched Nd: YAG laser, we obtained as much as $86 \%$ slope efficiency and $5 \times 10^{5}$ pulses lifetime. Furthermore, newly prepared deoxygenated samples exhibited even greater lifetimes.

Key words: Dye, xerogel, solid-state dye laser, stability, oxygen.

\section{Introduction}

During the past few years, much research has been devoted to solid-state dye lasers. Progress in host matrix processing has led to promising results, mainly with the doped solgel or the doped organic polymer techniques. ${ }^{1-5}$ We report a significant advance, more than 1 order of magnitude, toward stable solid-state dye laser emission. High conversion efficiencies were also obtained for these materials, and their synthesis is described. The latest results with deoxygenated samples indicate the likelihood of even better performance.

A xerogel, which can have a glassy aspect, is a solid gel obtained by gelification of a solution called sol, using the solgel process, which involves hydrolysis and polycondensation reactions.6,7 In some cases, these chemical reactions can be performed at room temperature. Any organic optically active molecule, especially a dye, can thus be incorporated into a sol in which it is soluble. The dopant molecules are trapped in the structure of the solid obtained by the sample synthesis, without being thermally destroyed in the process.

M. Faloss, M. Canva, P. Georges, and A. Brun are with the Groupe d'Optique Non Linéaire, Institut d'Optique Théorique et Appliquée, Centre National de la Recherche Scientifique, URA 14 Bâtiment 503, BP 147, 91403 Orsay Cedex, France. F. Chaput and J.-P. Boilot are with the Groupe de Chimie du Solide, Laboratoire de Physique de la Matiére Condensée, E'cole Polytechnique, Centre National de la Recherche Scientifique, Unité de Recherche Associée D-1254, 91128 Palaiseau Cedex, France.

Received 20 February 1996; revised manuscript received 27 January 1997.

0003-6935/97/276760-04\$10.00/0

(C) 1997 Optical Society of America
Many different doped xerogel samples were prepared from organically modified precursors (e.g., methyltriethoxysilane [MTEOS]). The method for general synthesis of silica-based sols and gels has been previously reported.1,6 A detailed description of the synthesis of each sample cannot be reported in this article, but will be given elsewhere. However, those parts common to all methods can be described. Hydrolysis of the silicon alkoxides was performed under acidic conditions with acetone as the common solvent. After several hours of hydrolysis at room temperature, a small amount of amine-modified silane (3-triethoxysilylpropylamine) was added to neutralize the acidity of the medium, thereby increasing the condensation reaction rate. An acetonic solution of Pyrromethene 597 purchased from Exciton (or other dopants such as Pyrromethene 567, Pyrromethene 580, Perylene Orange, or Perylene Red) was then added to yield a concentration in the range from $10^{-4}$ to $10^{-3} \mathrm{~mol} / 1$. The samples were overconcentrated, with respect to laser efficiency, to increase the operation lifetime by increasing the number of active molecules in the pumping volume. Afterward, the resulting sol was poured into polypropylene cylindrical-shaped molds and sealed.

Dense xerogel matrices were prepared to decrease scattering and optical inhomogeneities in the laser material. Thus we experimentally determined the drying conditions (time and temperature) leading to the most condensed MTEOS matrix by monitoring the NMR ${ }^{29} \mathrm{Si}$ signal. NMR spectra were recorded with a Bruker MSL 360 spectrometer with a Doty magic-angle-spinning (MAS) probe. The MAS spectra (spin of $4.5 \mathrm{MHz}$ ) were decomposed into lines to deduce relative contributions of the silicon atoms with 1,2 , or 3 siloxane bridges (Si-O-Si), which are 


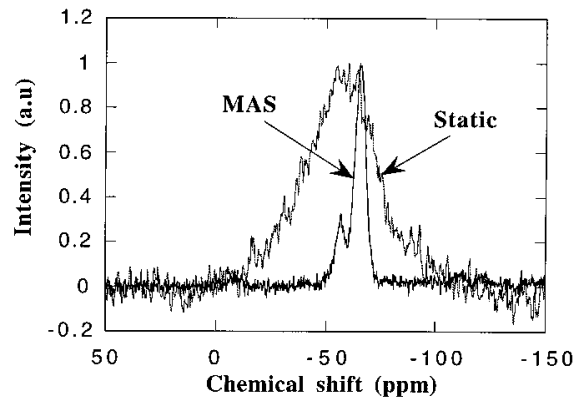

Fig. 1. ${ }^{29} \mathrm{Si}$ NMR spectrum of the dried MTEOS matrix (without and with MAS rotation).

conventionally denoted as $\mathrm{T}^{1}, \mathrm{~T}^{2}, \mathrm{~T}^{3}$. The progress of condensation reaction ( $\equiv \mathrm{Si}-\mathrm{OH}+\equiv \mathrm{Si}-\mathrm{OH} \rightarrow$ $\equiv \mathrm{Si}-\mathrm{O}-\mathrm{Si} \equiv)$ was characterized by the degree of condensation c, which was defined as

$$
c=\sum_{i} i q^{i} / 3
$$

where $q^{i}$ was the relative concentration of the $\mathrm{T}^{\mathrm{i}}$ species. Figure 1 shows the NMR spectra for the most condensed matrix we obtained after drying at $70^{\circ} \mathrm{C}$ for 45 days. The static spectrum exhibited a rigid-lattice signal showing the absence of a mobile sol phase. The MAS spectrum, which averaged the anisotropic interactions, presented two lines at -56.2 and $-65.6 \mathrm{ppm}$ (relative to the tetramethylsilane), corresponding to $\mathrm{T}^{2}$ and $\mathrm{T}^{3}$ species, respectively. A value of $c=0.94$ was deduced from the dried matrix, compared with $\sim 0.8$ just after gelation. After drying, optically clear and dense inorganic-organic hybrid xerogels $(30-\mathrm{mm}$ diameter and $15 \mathrm{~mm}$ thick) were obtained. Samples were hard enough to be machined and polished ( 4-nm roughness). They had good homogeneity, and their optical quality was very good with no scattering in volume. Figure 2 represents the transmission of an undoped xerogel, in the same order as transmission of a glass plate, which is also shown for comparison. Only Fresnel losses at the interfaces could be responsible for the decreased transmission.

It is known that dye-doped solutions are generally

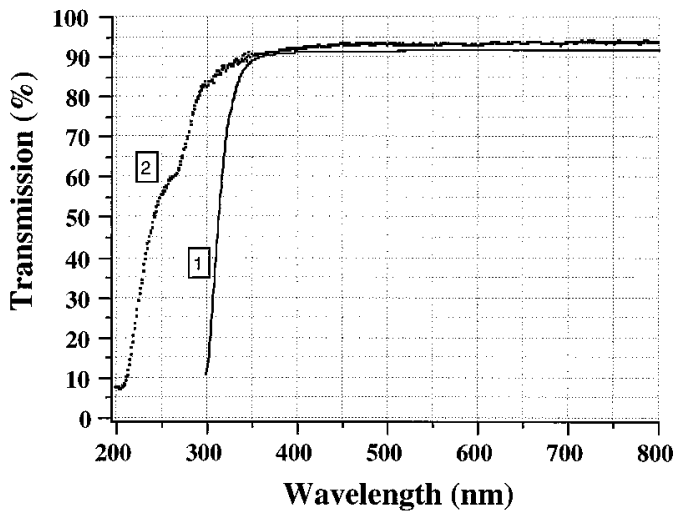

Fig. 2. Transmission of a glass plate (curve 1) and a MTEOSundoped xerogel (curve 2).

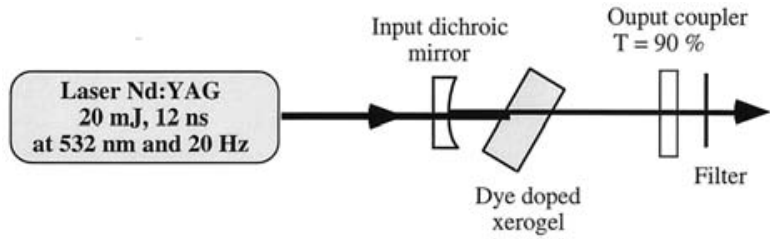

Fig. 3. Configuration of the laser cavity.

more stable in the absence of oxygen. In the case of xerogels, Rahn et al. ${ }^{3}$ achieved an initial gain of a factor of 4 for deoxygenated Pyrromethene 567-dopedsample lifetimes. We also prepared some oxygen-free samples. With regard to synthesis, all the manipulations were performed under $\mathrm{N}_{2}$ in a glove box and using Schlenk techniques. In this case, gelation and drying were longer than in the classical process, and 2 to 3 months were needed for sample preparation.

To study the samples, we used a 10-cm-long planoconcave linear cavity (Fig. 3). The flat output coupler transmitted 90\% energy in the 550-650-nm range. The concave mirror, with a $10-\mathrm{m}$ radius of curvature, was dichroic, with $R>80 \%$ in the 550 650-nm range, and $T=80 \%$ at $532 \mathrm{~nm}$. The sample was placed $\sim 1 \mathrm{~cm}$ from this input mirror and was optically pumped by a frequency-doubled Nd: YAG laser focused to an $800-\mu \mathrm{m}$-diameter spot on the sample. A colored filter (RG 550 from Schott) was used to filter the remaining nonabsorbed pump energy after the output coupler. The spatial profile of the pump beam was a flattop. The pump laser repetition rate was varied between single shot and $20 \mathrm{~Hz}$.

It appeared that the most efficient samples were obtained with the pyrromethenes (567, 580, and 597); which had 66\% to $86 \%$ slope efficiencies (Fig. 4). The perylenes led to around $55 \%$ for the orange specie and $20 \%$ for the red one.

The laser output beam profile, in a single-shot operation, was similar to that already reported with $\mathrm{M}^{2}$ values of $\sim 1.2 .{ }^{1}$

The tunability was obtained by introducing a prism in the cavity. Tunabilities of several tens of nanometers in several bands were obtained with the different

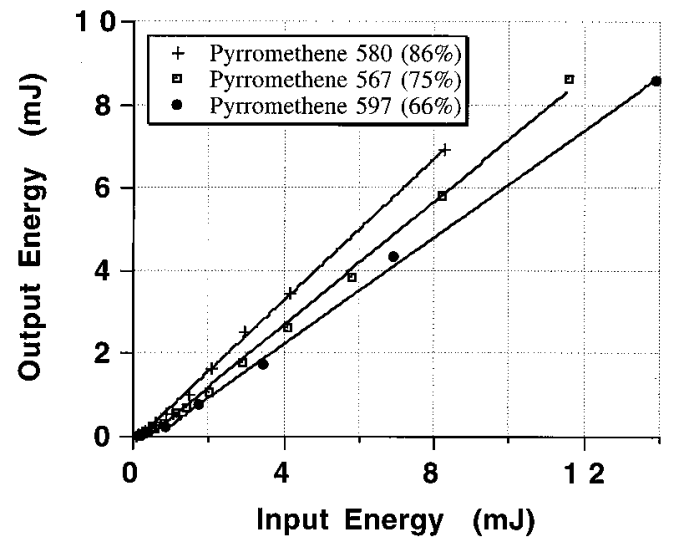

Fig. 4. Laser conversion efficiency (\%) with pyrromethene-doped samples. 
dyes (respectively, 565-605, 595-645, 547-585, 545595, and 570-625 nm, using Perylene Orange, Perylene Red, Pyrromethene 567, Pyrromethene 580, or Pyrromethene 597). These values were similar to those obtained in equivalent liquid solutions.

The most important result was in the comparatively high operating lifetime obtained at a relatively high repetition rate $(20 \mathrm{~Hz})$. To measure this, we recorded the evolution of the laser output energy as a function of number of previous sequentially emitted laser pulses. We defined a lifetime as the number of pulses that are emitted before output energy reaches half of its initial value, the latter corresponded to the output used to measure the conversion efficiency. The main reason for the decrease of the dye laser output was progressive destruction of the dye molecules caused by photodegradation and thermodegradation processes. Thus the lifetime was sensitive to the experimental setup and conditions, especially the energy and repetition rate of the pump source, but also its spatial profile. We generally used pump energy levels of $1 \mathrm{~mJ}$, and the repetition rate was varied to compare the relative importance of the two main dye degradation processes, photodegradation and thermodegradation, as analyzed in Ref. 8.

Using a pump energy of $1 \mathrm{~mJ}$ and a repetition rate of $20 \mathrm{~Hz}$, we obtained the most promising results with the Pyrromethene 597 dye trapped in hybrid matrices, $5 \times 10^{5}$ pulses. These results were nearly 1 order of magnitude greater than those we had previously obtained $\left(35 \times 10^{3}\right.$ pulses for the same Pyrromethene 597 at 1 -mJ pump energy and $20-\mathrm{Hz}$ repetition rate). ${ }^{1}$

For some applications, as in chemistry, biology, or medicine, typical energy required is $\sim 1 \mathrm{~mJ}$. Thus we recorded as a function of time the evolution of the laser output energy starting from $1 \mathrm{~mJ}$. This way of measuring the lifetime for identical output energy leads to comparisons in terms of performance, rather than stability, as for identical input energy.

At a $20-\mathrm{Hz}$ repetition rate, the best results were obtained with a Pyrromethene 597-doped xerogel, $12 \times 10^{4}$ pulses (Fig. 5). To provide an initial output of $1 \mathrm{~mJ}$, this sample, of slightly less efficiency than the one shown in Fig. 1, was pumped at $\sim 3 \mathrm{~mJ}$. It was thus submitted to a fluence of around $0.6 \mathrm{~J} / \mathrm{cm}^{2}$ or an average power surface density of $12 \mathrm{~W} / \mathrm{cm}^{2}$.

These great improvements in solid-state dye laser performance over the past years are a result of simultaneous optimization of sample synthesis, the laser cavity, and the pump laser. They are, to the best of our knowledge, the longest lifetimes obtained in xerogel systems and are comparable with the latest published results obtained with plastics, if used in similar conditions.

In the case of perylenes, the laser cannot be operated at such a high repetition rate if we wish to maintain relatively long lifetimes. This result clearly indicates that pyrromethene-doped samples are less sensitive to thermal degradation than perylene-doped samples. It can however be noted that, since the energy conversion efficiencies are higher for the former than the latter, less energy is deposited in the sample, and the local temperature is thus lower.

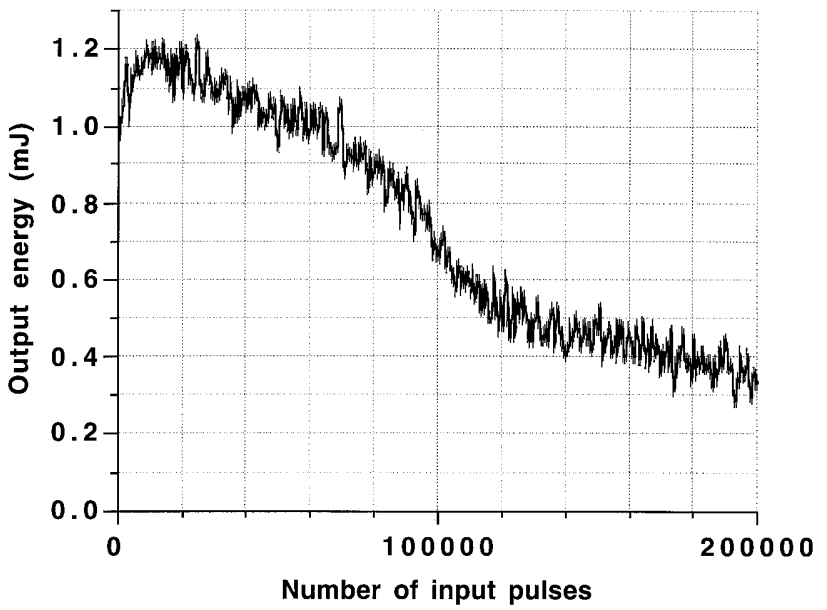

Fig. 5. Output energy evolution as a function of a previously emitted pulse. The initial output energy was $1 \mathrm{~mJ} /$ pulse, and the laser was operated at $20 \mathrm{~Hz}$. The xerogel was doped with Pyrromethene 597.

In a commercial laser the dye-doped liquid or solidgrain medium is continuously moved. This uses more of the volume of the active medium and thus increases the lifetime, considering that the total number of active molecules is increased. This movement also decreases the repetition rate at which each area is used, thus decreasing the thermal problems.

To illustrate this point with our samples, we used two translation stages (operating in two orthogonal directions), which automatically scanned the gain medium in a square of $8 \times 8 \mathrm{~mm}^{2}$ at a speed of 0.3 $\mathrm{mm} / \mathrm{s}$, which represents only a small part of the sample. Because we used an area of $64 \mathrm{~mm}^{2}$ instead of a unique zone of $800-\mu \mathrm{m}$ diameter, we should have gained at least 2 orders of magnitude in lifetime. As expected, such a solid-state dye laser system exhibited a stable daily output during the few weeks we conducted the experiment, emitting more than $12 \times$ $10^{6}$ pulses. This result indicates that the laser system could operate at $\sim 1 \mathrm{~mJ}$ of output energy at $20 \mathrm{~Hz}$ with a single sample for several months. Operating the laser $8 \mathrm{~h}$ a day at $20 \mathrm{~Hz}$, it took more than 20 working days to emit these $12 \times 10^{6}$ pulses.

All the performances reported above are stable with time, even if some samples seem to improve with aging, normally stored in an opaque box at room temperature. However, during this study, we were forced to pay attention to many different parameters of the solgel synthesis that, while not crucial, are relatively important; these were partially responsible for the increase in performance. In particular, the drying temperature and duration were much more important than is commonly thought. Having to avoid dye thermal degradation and dye aggregation, we concluded that these synthesis parameters govern the monomeric dye xerogel concentration. In this respect, the values given in the part relative to the sample synthesis are only indicative. Current experiments are being conducted to study these effects quantitatively and will be reported in due time. With regard to the op- 


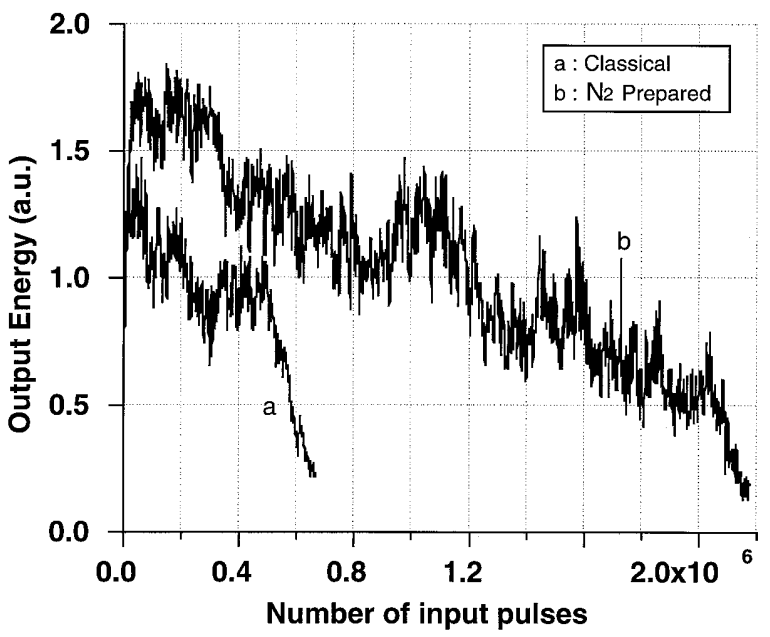

Fig. 6. Output energy evolution of the laser with a Pyrromethene 597-doped sample prepared in classical conditions (curve a) and in oxygen-free conditions (curve b). The pumping conditions were 1 $\mathrm{mJ} /$ pulse at $20 \mathrm{~Hz}$.

tical setup modification, compared with previously published research, ${ }^{1}$ we have evidence that the use of a flattop laser, which more homogeneously utilizes the sample pumped areas, is also part of the reason for the longer lifetimes now achieved.

With respect to lifetime, the sample synthesis can still be optimized, as we show below.

Indeed, preliminary results obtained with the most recently synthesized samples prepared in oxygenfree conditions have been very promising.

With a Pyrromethene 597-doped sample (concentration, $5.5 \times 10^{-4} \mathrm{~mol} / \mathrm{l}$; thickness, $1 \mathrm{~cm}$ ), we obtained well over $1 \times 10^{6}$ pulses at $1 \mathrm{~mJ} /$ pulse and $20-\mathrm{Hz}$ pumping conditions (Fig. 6). With this sample, we measured a slope efficiency of $40 \%$. The fact that the cavity was operated close to the threshold probably accounts for the instability of the laser output.

With the Perylene Red-doped sample, 1-mJ/pulse pumping energy, we obtained $\sim 2 \times 10^{5}$ pulses at $10 \mathrm{~Hz}$ and $2.5 \times 10^{5}$ at $5 \mathrm{~Hz}$; thermal problems prevented operation at $20 \mathrm{~Hz}$. These values compare favorably with the $5 \times 10^{3}$ and $1 \times 10^{4}$ pulses, respectively, obtained for similar samples prepared in normal conditions. These values, although lower than those for pyrromethene, indicate a much more dramatic effect of preparing the samples in oxygen-free conditions.

However, when the samples were stored in air, these lifetime characteristics decreased during the following days, and the slope efficiency tended to increase slightly. We attribute this effect to oxygen diffusing into the samples.

This led us to try to remove the oxygen from the matrices. This was achieved by placing some samples into a vacuum chamber for a few hours and then returning them to a nitrogen atmosphere for a few days. All the pyrromethene-doped samples gained a factor of between 2 and 5 in lifetimes with only a slight decrease $(\sim 10 \%)$ in their conversion efficiency.

These new results clearly show the interesting pos- sibilities of deoxygenating the perylene- and pyrromethene-doped samples, which is in good agreement with previous results in dye-doped solutions and solids. Oxygen reacts with excited molecules in the triplet state, leading to dye molecule destruction. However, these findings still need to be clarified because it is also well known that oxygen can act as a quencher of the triplet state that protects the dye molecules from destruction. In our case, the first effect seems to have been predominant. These results in solgel materials are comparable with the latest good lifetime performances obtained with doped polymers. ${ }^{5}$ Further investigation of this type of synthesisproducing oxygen-free samples is clearly warranted.

In conclusion, we now routinely prepare chemically stable materials that achieve $\sim 80 \%$ conversion efficiency, large tunability, and a lifetime of $\sim 5 \times 10^{5}$ pulses when operated at a millijoule energy level and at $20 \mathrm{~Hz}$. Current investigations with deoxygenated samples show that 1 order of magnitude can be gained in operating lifetimes, an increase of well over $1 \times 10^{6}$ pulses. The conversion efficiency of pyrromethenes is attractive. However special attention must be paid to the perylenes that appear to possess, if thermodegradation can be minimized, a higher potential effect on lifetimes in terms of photostability. ${ }^{8}$ However in the case of these perylene dyes, we still have to enhance the energy conversion efficiencies and increase the matrix thermal conductivity.

Altogether, these results are promising and indicate that effective tunable solid-state dye lasers will replace liquid-dye laser systems in certain applications. With only one sample and emitting approximately a millijoule, the lifetime of such a system, in which the gain medium is moved, can be increased to several months.

We thank Yann Lechantre and Gilles Colas from the Institud d'Optique for adequate sample optical polishing.

\section{References}

1. M. Canva, P. Georges, J. F. Perelgritz, A. Brun, F. Chaput, and J. P. Boilot, "Perylene- and pyrromethene-doped xerogel for a pulsed laser," Appl. Opt. 34, 428-431 (1995).

2. B. Dunn, F. Nishida, R. Toda, J. Zink, T. Allik, S. Chandra, and J. Hutchinson, “Advances in dye-doped sol-gel lasers," Mat. Res. Soc. Symp. Proc. 329, 267-277 (1994).

3. M. D. Rahn, T. A. King, C. A. Capozzi and C. A. Seddon, "Characteristics of dye doped ormosil lasers," in Proc. SPIE 2288, 364 371 and 382-391, J. D. Mackenzie, ed., Sol-Gel Optics III, (1994).

4. R. Reisfeld, "Film and bulk tunable lasers in the visible," in Sol-Gel Optics III, J. D. Mackenzie, ed., Proc. SPIE 2288, 563$572,(1994)$

5. S. Chandra and T. H. Allik, "Compact, high-brightness solidstate dye laser," Adv. Solid-State Lasers 24, 119-122 (1995).

6. F. Devreux, J. P. Boilot, F. Chaput, and A. Lecompte, "Sol-gel condensation of silicon alkoxides," Phys. Rev. A 41, 6901-6909 (1990).

7. C. J. Brinker and G. W. Scherer, Sol Gel Science, The Physics and Chemistry of Sol Gel Processing, (Academic, New York, 1990).

8. A. Dubois, M. Canva, A. Brun, F. Chaput, and J. P. Boilot, "Photostability of dye molecules trapped in solid matrices," Appl. Opt. 35, 3193-3199 (1996). 